\title{
Cloud Computing based Real Time Monitored Supply Chain System
}

\author{
Usama Zafar Ansari' ${ }^{1}$, Zheng Liu ${ }^{2}$ \\ ${ }^{1}$ School of Engineering, Faculty of Applied Science \\ University of British Columbia, Okanagan Campus, Kelowna, BC, Canada - V1V 1V7 \\ usama101@mail.ubc.ca \\ ${ }^{2}$ School of Engineering, Faculty of Applied Science \\ University of British Columbia, Okanagan Campus, Kelowna, BC, Canada - V1V 1V7 \\ zheng.liu@ubc.ca
}

\section{Extended Abstract}

The supply chain monitored in the real-time using the sensors is needed to make supply chain flow efficient. The COVID19 pandemic has added pressure on to the federal and provincial government to identify appropriate vaccine distribution solutions. The World Health Organisation estimates that more than $50 \%$ of vaccines may be wasted globally every year because of temperature control, logistics and shipment-related issues [1]. The Internet of Things (IOT) sensors are widely used in reporting the sensors data in terms of temperature sensors, humidity sensors, location sensors, pressure sensors etc... In order to reduce the losses of the vaccines throughout the supply chain, such a solution was needed to allow the cold supply chain stakeholders to take action if the temperature or humidity of a batch of vaccines begin to exceed or reduce its required level and also to allow the stakeholders to view the location of a specific batch of vaccines while in transit. There has been a great loss of vaccines in the transit which is resulting into an expensive overhead for the supply chain.

So, there is a need of real-time monitoring of vaccine shipment using Internet of Things (IOT) sensors embedded into the shipping container to track the location, temperature, and humidity of vaccine batches during the transportation [2]. Since these sensitive properties will be monitored in a real time which would ultimately lead to a fewer loss of the vaccines. As the vaccines are temperature and humidity dependent, slight change in these properties range can make the vaccines inefficient to use or even harmful to the person they are injected to. So, real-time monitoring of vaccines in the complete supply chain process is required so that vaccines reaching the end of the supply chain is efficient and in good condition to be used for vaccination. The data of the effective supply chain needs to be trusted and immutable, this makes the transfer of ownership of the vaccine in supply chain trustworthy.

Application of cloud computing framework to create the IOT monitored real-time supply chain based system resolve the problem. The system handles the IOT sensor data consisting of temperature, humidity, location, date, time and sensor id to create the effective flow to handle the IOT sensor data. These sensor data is linked to the supply chain containers associated with the vaccines to be transported. The supply chain process flow is executed to build the trust on data. The use of Amazon Quantum Ledger Database, which is an immutable and cryptographically verifiable database is used to execute the supply chain transactions and ownership transfer of vaccine container in the supply chain flow [3]. As the supply chain process expands the ledger database adapt to the scalability, so this becomes compatible to increase and decrease of load. The cloud computing based architecture interact with the different services to execute the supply chain flow. The secure onboarding of all the stakeholders involved in supply chain is the first step. Then it leads to manufacturer adding the product in the supply chain for the distributer to place the order. Once the order is placed then it leads to $3^{\text {rd }}$ party logistic involved in the supply chain to transport the placed order in the secured and real-time monitored way. The sensor data coming from the transported container, is monitored on the dashboard. The stakeholders are notified if the temperature or humidity of the container is increased or decreased from their threshold. This notification can help them to take timely action to prevent damage or if it is exposed for longer time then it becomes inefficient to use. This cloud-based system helps in functioning of all the elements involved in supply chain in effective manner. This system will help in future to integrate other cloud services like chat bots, machine learning predictions, supply chain bills management etc... to expand accordingly to accommodate needs to effective working of supply chain flow. 


\section{References}

[1] J. Thomas. (2018, Jul 24). Over half of vaccines are wasted globally for these simple reasons [Online]. Available: https://www.weforum.org/agenda/2018/07/the-biggest-hurdle-to-universal-vaccination-might-just-be-a-fridge

[2] Zhang, Y., Chen, B., \& Lu, X. (2011, August). "Intelligent monitoring system on refrigerator trucks based on the internet of things" in International Conference on Wireless Communications and Applications (pp. 201-206). Springer, Berlin, Heidelberg.

[3] A. Certain. (2019, Dec 23). In Data We Trust: The Why And How Of Amazon QLDB, A Purpose-Built, Immutable And Verifiable Database [Online]. Available: https://www.forbes.com/sites/amazonwebservices/2019/12/23/in-datawe-trust-the-why-and-how-of-amazon-qldb-a-purpose-built-immutable-and-verifiable-database/?sh=d81654651c7b 